Neurotherapy is a collection of non-invasive approaches to changing the way the brain functions, which combine the concepts of learning theory and neuroplasticity. While the applicability of neurotherapy to the treatment of clinical conditions ${ }^{1,2} 3$ might initially imply little relevance to the development of healthcare leaders, this is not the case. In addition to helping leaders overcome a number of cognitive challenges which can accompany "executive stress" (discussed in Hartney, ${ }^{4}$ this issue), such as difficulty with concentration and focus, ${ }^{5}$ poor sleep quality, ${ }^{6}$ anxiety, depression, ${ }^{7}$ and substance use issues, ${ }^{8}$ neurotherapy can be used to help maximize leadership potential through peak performance training, involving enhancement of creativity, cognitive efficiency, and executive functioning. ${ }^{9}$

It is important to understand that neurotherapy is a form of training, that is used to enhance the skill of the person in using his or her brain more efficiently. Just as physical exercise can greatly benefit and enhance the physical performance of healthy individuals, in addition to those with physical health conditions, neurotherapy can facilitate healthcare managers to function more effectively, whether or not they have cognitive impairments. There are parallels with other forms of training; consistent practice is required for improvements to occur, and, just as working out regularly at the gym will consistently improve strength and stamina, but will not make everyone an Olympic athlete, the benefits of neurotherapy, while substantial, will be limited to the scope of the individual's potential, and capabilities, and mastery.

Neurotherapy is based on knowledge that the brain can change, functionally, so that neurological conditions associated with specific behaviors can be altered through learning and neuroplasticity, ${ }^{10}$ thereby modifying clinical disorders, academic and creative performance, and leadership effectiveness. While behavioural approaches, such as practicing mindfulness meditation, will help with many cognitive challenges faced by healthcare leaders, ${ }^{5}$ in more challenging situations, neurotherapy will produce a vastly accelerated and specifically targeted improvement in performance and mental functioning.

There are two forms of neurotherapy; both focus on teaching the individual to gain control over the brainwave frequencies associated with various mental states or functions. Figure 1 provides an overview of the main brainwave frequency bands used in neurotherapy. 


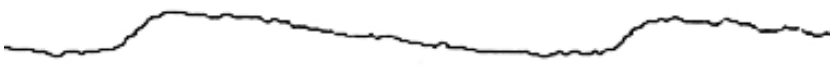

Delta waves are slow and predominate during sleep

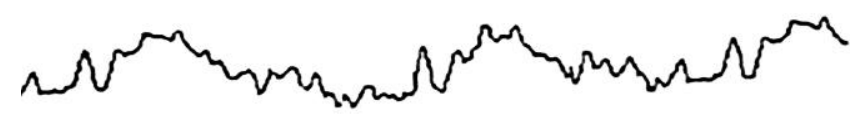

Theta waves predominate when inattentive or deeply relaxed

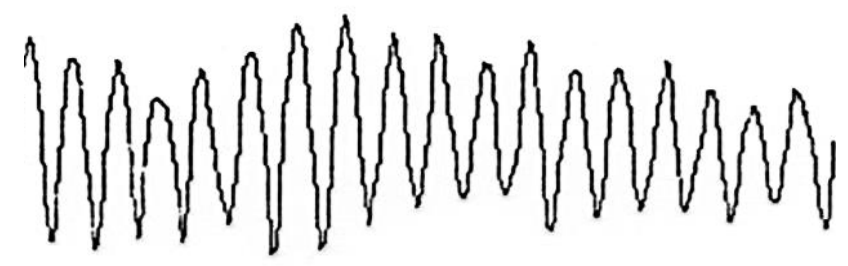

Alpha waves predominate during creativity and alert relaxation

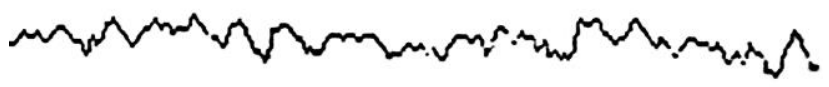

Beta waves predominate during focused concentration and high attentiveness

Figure 1. Brainwave frequency bands and descriptions.

Neurofeedback, or brainwave biofeedback, uses an operant conditioning approach to teaching control of brainwave activity. This training approach involves providing feedback to the individual about his or her brain activity, thereby reinforcing positive change in mental state. When the brain is performing according to targets agreed with the neurotherapist, the individual will receive feedback, typically in the form of a pleasing tone or visual image displayed on the computer monitor. Through repetitive training, this feedback guides the person to positively influence, and ultimately, to gain control over her or his brain functioning, in the same way that 
repetitive training at the gym will strengthen one's control over the physical functioning of the body.

"Braindriving” technologies ${ }^{11}$ use various forms of stimulation, including light, electromagnetic, and electrical current to influence brain state. This is based on the classical conditioning learning theory, which readers may be familiar with through the well-known research by Pavlov. The classical conditioning of brainwave activity was demonstrated in the 1940s. ${ }^{12}$ The treatment involves presenting an unconditioned stimulus, conditional on the individual learning to change in the amplitude of a particular brainwave range, associated with the targeted area of functioning. For example, lights flashing at $16 \mathrm{~Hz}$ will suppress the amplitude of alpha range brainwaves. If the purpose of treatment is to learn to suppress alpha, then every time alpha amplitude crosses a training threshold, the flashing lights are turned on until alpha amplitude drops below the threshold. This assists the individual in learning how to initially access desired mental states.

There are two important ways to arrive at training protocols. The first is to identify and correct anomalies in the person's EEG that are not associated with leadership prowess, such as anxiety, forgetfulness, or inattention; the second is to teach leaders how to produce brainwave patterns that are associated with the characteristics of effective leadership, for example, stress tolerance, cognitive processing proficiency, brain efficiency, and executive functioning, so that eventually, they can independently access these highly functional states in the workplace.

The application methodologies vary, ranging from one-size-fits-all protocols and symptom driven protocols, to protocols driven by neurological assessment (Quantitative Electroencephalogram - QEEG) to identify areas of inefficiency and those associated with predisposition to specific conditions, such as depression. As psychologists, we prefer the latter, more individualized approach, whereby the brain is assessed, anomalous patterns are identified, and the individual is taught to bring their brainwaves into the desired range. Once the brain “platform” is standardized, this is followed by neurotherapy to teach the person how to optimize their own brain functioning.

To assess irregular brain activity, we need individualized data to help us determine brain activity that is anomalous. But how do we determine “normal?” One method is to select individuals with no reported clinical conditions for a number of years, and then use that group as the "normal” group against which to compare a subject's brain activity. This is the typical 
approach used in psychological assessment, but it is problematic for a number of reasons, not least of which, the logic is wrong. Aside from skepticism about what constitutes "normality,”13 and particularly for leaders, who may fall outside of the norm in various ways, such normative databases do not identify predispositions. Conversely, clinical databases, as the name implies, are databases containing clinical data on clients who have presented themselves for treatment of problems. The clients' self-reported symptoms are then matched with their brain data, so patterns are discerned associated with these conditions. It is important to keep in mind that non-clinical subjects may well have non-manifested predispositions to a condition, but not be symptomatic. Furthermore, databases of "normal" individuals may be statistically blind to genetic predispositions.

Let us take the case of optimizing the executive brain. In elite athletic competitions, it is not uncommon for the winner to be ahead by less than a second or less than a half point on a rated event. So "optimizing" in this athletic arena may be an increase in performance by one or two percent. Thus, we are not talking about "better” but rather "optimal.” Optimizing neurotherapeutic treatment focuses on facilitating optimal performance of a person's brain, by orchestrating the complex interplay between different brain functions, some of which may, in fact, best remain outside of the normal range, particularly if the individual is highly creative, or is gifted in mental focus.

In the case of IQ, the window for improvement (increasing IQ) is in the range of 15 IQ points. ${ }^{14}$ Critically, it has been found that this window is fairly consistent, regardless of the person's baseline IQ. In other words, borderline IQ has the same window as superior IQ. The lower the baseline, the greater the percentages; whereas a 15 IQ point increase for borderline would be about $21 \%$ improvement, the same increase for superior would be about $12 \%$.

Healthcare leaders should note that the "optimal performance" concept, then, can be applied to all healthcare employees. One can expect to achieve increased brain efficiency, relative to the individual's baseline. An intellectually limited individual (e.g., "borderline”), for example, may be elevated to "low average" functioning, allowing him or her to proceed to employment and independent living. Similarly, a person on the autism spectrum may acquire more appropriate social skills after improvements in brain efficiency, and in turn obtain employment. Such situations represent an optimizing of brain functioning within the window of functional change attainable from baselines. Whereas historically, people with such cognitive 
challenges would be excluded from the workforce, today, leaders whose values support employment of individuals across the range of cognitive abilities, who embrace diversity, recognize the strength it brings to their teams, and who wish to be inclusive of disadvantaged and patient populations within their workforce, have a new tool in neurotherapy to facilitate inclusive employment.

One final consideration with regard to the concept of "optimization" relates to the stability of results. For most conditions, and certainly for Optimal Performance training, the best metaphor for the stabilization issue is going to the gym for physical training. Once your body is in good shape, you don't abandon your exercise maintenance program and sit on a sofa and eat potato chips. A person treated for poor stress tolerance, for example, would be put on a homebased maintenance program, to stabilize the neurological changes achieved with neurotherapy.

Neurotherapy holds great promise for the aging population of healthcare leaders. Older employees being treated for age-related declines in brain efficiency should not expect stability, but rather, that continuous treatment is required to mitigate, such age related declines, to the highest extent possible. ${ }^{15}$ Hence, for optimal performance of mature leaders, one would expect ongoing treatment to maintain the optimization, although the ongoing maintenance training would not require the same intensity of training once optimization has been achieved.

A concern often expressed by people with unique talents is that brainwave training may diminish their skills. The one-size-fits-all neurofeedback programs that have been developed claim to normalize brain activity. Yet, if I happen to be an exceptional leader, I don't want to have my brain normalized. Optimizing brain activity can involve training areas of the brain to be outside of normative ranges. An example is activity of the anterior cingulate gyrus (ACG). This subcortical structure sits below the frontal midline of the brain and when activity of this area is elevated, clients often report obsessive/compulsive like behaviors, such as perseverative thoughts. If one perseverates on depressive thoughts, then depression is deepened and more resistant to treatment. However, if negative thoughts are not present, then the elevated activity is related to perseverance such that the leader can become "fixated" and "obsessive" about solving problems or creating strategies; this is a constructive and highly functional outcome to what, in another individual, might be a problematic state.

Obtaining a "brain map" to identify areas of brain inefficiencies associated with cognitive and emotional problems (see Swingle ${ }^{16}$ for details of this procedure) allows an 
individualized approach to neurotherapy, rather than a one-size-fits all. The assessment is brief and remarkably accurate. The brain assessment (ClinicalQ) requires about 6 minutes of recording time, and is the first step in the optimal performance procedure. So, for example, if the client has brain markers for predisposition to depressed mood states or poor stress tolerance, those conditions are addressed first. After normalizing these problematic predispositions, then the individual is ready for optimal performance training focused on maximizing brain efficiency, base arousal levels and perseverance.

By way of illustration, the ClinicalQ is described in the following case vignette, along with the protocols for enhancing leadership performance.

\section{The Case of Martin}

Martin was a successful, mid-career health director, who had quickly risen to a senior position following his leadership of an ambitious strategic plan to streamline a number of health services related to mental health and substance use. He was applauded by his superiors for an elegant model of service that had apparently translated into savings of millions of dollars for the health authority. After being put in charge of operationalizing the plan, Martin's career took a nosedive. Complaints from staff and the community were soon picked up by the media, causing embarrassment for Martin and his senior colleagues. He attempted to cope with the resulting difficulty he had relaxing with a combination of alcohol and occasional sleep medication, which, to his surprise, seemed to make him even more anxious during the day. Eventually, a high profile patient death, apparently resulting from the patient not receiving essential, urgent services as a direct consequence of Martin's service cuts, sent him over the edge. Unable to cope with workrelated stress, Martin took an extended period of sick leave, during which he presented for treatment.

The results of Martin’s ClinicalQ indicated several problematic areas. His brain map indicated a "traumatized artist" signature - elevated alpha waves at the back of the brain (O1), which were quickly suppressed. This is common among people who are highly creative, yet have experienced traumatic stress in the recent or distant past. Also at the back of the brain (O1), there were excessive beta waves (16-25 Hz), and suppressed theta waves (3-7 Hz), indicative of anxiety and insomnia. Finally, the ratio of high alpha (11-12 Hz) to low alpha waves (8-10 Hz) 
centrally $(\mathrm{Cz})$ indicated that Martin’s cognitive processing had become inefficient, likely due to his use of alcohol and sleep medication. Symptomatically, elevation of fast frequency or gamma (26-40 Hz) amplitude over the ACG (Cz) is associated with perseverative thought processes.

Consistent with these observations, Martin complained that his once visionary leadership had all but disappeared; he was unable to think clearly, he had become forgetful, and his thought processes were either foggy, or excessively anxious. He found it difficult to sleep, due to his brain being "too busy."

The first step in the optimal performance training was for Martin to gain control over the above described inefficiencies. This was accomplished with neurotherapy, as described in the introduction, and then Martin was able to commence the optimizing portion of his training program.

Neurofeedback focused first on quieting Martin's mind by teaching him to reduce the excessive beta waves at the back of the brain (O1), and then addressing the trauma response by training his alpha waves to rise with his eyes closed. Martin had a few nights of troubled sleep, but found that quite quickly, he began to sleep more deeply, his anxiety was under control, and his memory became sharper. Then he worked on regaining optimal cognitive performance through increasing his high alpha waves in relation to his low alpha waves. His memory improved further, and he found he was able to visualize in the way he could prior to experiencing work-related stress, and to focus deeply and extensively on problem-solving, while no longer becoming excessively anxious. 


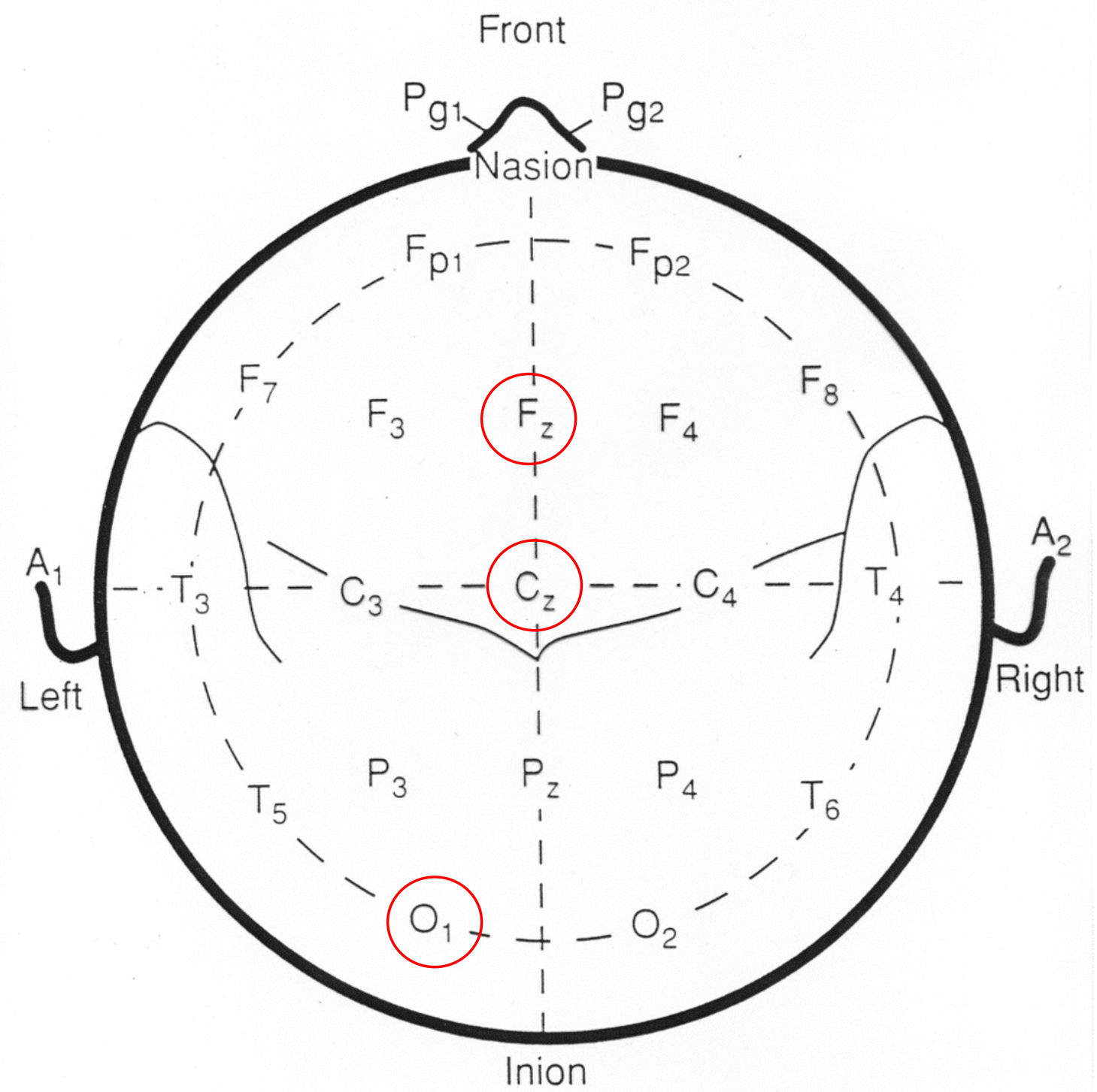

Figure 2. 10-20 international brain site schematic illustrating Martin’s ClinicalQ.

The optimal performance aspect of training included increasing brain efficiency by increasing Alpha Peak Frequency (APF). APF is the average of the highest peaks in the alpha (8$12 \mathrm{~Hz}$ ) range. The faster the APF, the higher the IQ, with related improvements in cognitive functioning as well as improved immune system functioning $\cdot{ }^{17}$ In addition to accelerating alpha frequency, Martin learned to increase alpha amplitude at locations $\mathrm{O} 1$ and $\mathrm{Cz}$, to enhance his visualization skills, and reduce the lag time between changes in state, such as between eyes open and eyes closed conditions. As the negative emotional conditions resulting from Martin's trauma 
were mitigated by normalizing the functioning in the occipital region, Martin decided to leave the ACG "hot" to facilitate the willfulness and determination factors.

On measures of his presenting symptoms (e.g., sleep problems, worrying, reduced cognitive effectiveness, depressed mood states and irritability) Martin improved substantially in all these areas. He reported being very much more effective cognitively in his leadership functions.

With a clearer mind, Martin found it easier to reflect on what had gone wrong. He realized firstly, that he needed to complement his own visionary abilities with the skills of others in healthcare operations, and secondly, that he needed to develop a more meaningful connection with the community he served, through consulting with community-based teams. With his anxiety under control, he found it easier to assert himself with his senior leadership team, and advocated for a collaborative partnership with colleagues who had skills which were complementary to his own. He now takes time for self-care, regularly practicing mindfulness meditation, and, due to being confronted with his own vulnerability, is now better able to empathize with, and meet the needs of the people his organization serves.

As in Martin's case, the optimal performance regimen for employees who are at risk of experiencing traumatic stress through their work should recognize several brain features that would make them more vulnerable to being disabled by severe stress and burnout. Employees who show the markers for exposure to traumatic level stress also show marked deficiency in the theta/beta ratio in the occipital region of the brain, which is associated with poor stress tolerance. ${ }^{18}$ So, rather than treating an employee after exposure to traumatic stress, it is much more efficient to make the brain more stress tolerant before the employee is exposed to a stressful work environment. Therefore, had Martin been given theta/beta treatment prior to experiencing stress, he is likely to have been better able to cope, and therefore, could have led the organization more mindfully, perhaps avoiding the triggering traumatic event, and the catastrophic outcomes for the community, patient, and patient's family, as well as himself. This is analogous to strength training to avoid a workplace injury, if one's work responsibilities include heavy lifting.

The preparation for duty concept, in which stress tolerance is enhanced prior to engagement/exposure to acute stress, is designed to reduce stress-induced mental and/or physical health difficulties. Increasing stress tolerance also improves decision making under highly 
stressful conditions, and it has been long known that hyper-arousal is associated with declines in

executive functioning. ${ }^{19} \mathrm{~A}$ most interesting randomized controlled study of biofeedback training in crisis managers, with many similarities to the case of Martin can be found in the study by Janka and colleagues. ${ }^{20}$

\section{Conclusion}

In conclusion, neurotherapy is an underutilized approach to enhancing the development of leadership skills by training one's own cognitive efficiency. It has specific applicability to managing "executive stress" while leading in the complex environment of healthcare. By modifying one's own brain performance through responding to feedback on brainwave frequencies, leaders can overcome challenges to cognitive effectiveness resulting from personal tendencies or reactions to stress, and can optimize their performance through improved executive functioning and mental clarity. While neurotherapy requires active engagement on the part of the individual leader, in combination with self-reflection, it can be valuable approach to addressing cognitive challenges to leadership functioning.

1 Jensen MP, Gertz KJ, Kupper AE, Braden AL, Howe JD, Hakimian S, Sherlin LH. Steps toward developing an EEG biofeedback treatment for chronic pain. Appl Psychophysiol Biofeedback. 2014; 38:101-108.

2 Alves-Pinto A, Turova V, Blumenstein T, Hantuschke C, Lampe R. Implicit learning of a finger motor sequence by patients with cerebral palsy after neurofeedback. Appl Psychophysiol Biofeedback, 2017; 42:27-37.

3 Koberda JL. Traumatic brain injury: Is neurofeedback the best available therapy? J Neurol Neurobiol. 2015; 1(3). doi http://dx.doi.org/10.16966/2379-7150.110

4 Hartney E. (this issue). A three step model of stress management for healthcare leaders. Healthcare Management Forum.

5 Mohapel P. (this issue). The neurobiology of focus and distraction: the case for incorporating mindfulness into leadership. Healthcare Management Forum. 6 Cortoos A, De Valck E, Arns M, Cluydts R. An exploratory study on the effects of teleneurofeedback and tele-biofeedback on objective and subjective sleep in patients with primary insomnia. Appl Psychophysiol Biofeedback. 2010; 35:125-134.

7 Linden DEJ. Neurofeedback and networks of depression. Dialogues Clin Neurosci. 2014; 16(1): 103-112.

8 Lackner N, Unterrainer HF, Skliris D, Wood G, Wallner-Liebmann SJ, Neuper C, Gruzelier $\mathrm{JH}$. The effectiveness of visual short-time neurofeedback on brain activity and clinical characteristics in alcohol use disorders: Practical issues and results. Clinical EEG and Neuroscience. 2016;47(3):188-195. 
9 Ford NC, Sherlin LH. Peak performance and neurofeedback: Growing popularity of neurofeedback for peak performance training. NeuroConnections. 2016; 1-10.

10 LeDoux J. Synthetic self: How our brains become who we are. 2002; New York, NY: Viking. 11 Swingle PG. Biofeedback for the Brain. 2010; Piscataway, NY: Rutgers University Press. 12 Jasper H, Shagass C. Conditioning of the occipital alpha rhythm in man. Journal of Experimental Psychology. 1941; 28(5):373.

13 Olding S. Pathologies: A life in essay. 2008; Calgary, AB: Freehand Books.

14 Tansey MA. Wechsler (WISC-R) changes following treatment of learning disabilities via EEG biofeedback training in a private practice setting. Australian Journal of Psychology. 1991; 43(3):147-153.

15 Budzynski T, Budzynski HK, Tang H-Y. Brain brightening: Restoring the aging mind. In: Evans JR. Handbook of Neurofeedback. Binghampton, NY: Haworth Medical Press; 2007;231265.

16 Swingle PG. Adding Neurotherapy to Your Practice. 2015; New York, NY: Springer. 17 Arns M. EEG-based personalized medicine in ADHD: Individual alpha peak frequency as an endophenotype associated with nonresponse. Journal of Neurotherapy, 2012;16:123-141. 18 Swingle PG. The effects of negative emotional stimuli on alpha blunting. Journal of Neurotherapy. 2013;17(2):133-138.

19 McClelland, D. Human Motivation. 2000; Cambridge UK: Cambridge University Press. 20 Janka A, Adler C, Brunner B, Oppenrieder S, Duschek S. Biofeedback training in crisis managers: A randomized controlled trial. Appl Psychophysiol Biofeedback, 2017; 42:117-125. 